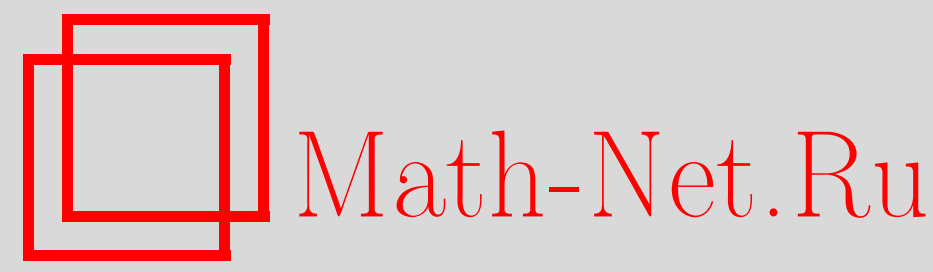

И. В. Филимонова, Асимптотика решений полулинейного параболического уравнения в цилиндрической области, Матем. сб., 2004, том 195, номер 2, 141-156

DOI: https://doi.org/10.4213/sm803

Использование Общероссийского математического портала Math-Net.Ru подразумевает, что вы прочитали и согласны с пользовательским соглашением http://www . mathnet.ru/rus/agreement

Параметры загрузки:

IP: 54.209 .52 .79

26 апреля 2023 г., 13:08:33 
УДК 517.95

\author{
И.В. Филимонова
}

\title{
Асимптотика решений полулинейного параболического уравнения в цилиндрической области
}

\footnotetext{
В работе найдена асимптотика при $t \rightarrow \infty$ решений полулинейного параболического уравнения второго порядка в цилиндрической области, удовлетворяющих нулевому граничному условию Неймана на боковой поверхности цилиндрической области.

Библиограф̆ия: 8 названий.
}

\section{§1. Введение}

В настоящей работе рассматривается полулинейное параболическое уравнение

$$
u_{t}=\Delta u-a(x)|u|^{\sigma} u
$$

в цилиндре $\Pi_{0}=\{(x, t) \mid x \in \Omega, t>0\}$, где $x=\left(x_{1}, \ldots, x_{n}\right), \Omega$ - ограниченная область в $\mathbb{R}^{n}$ с гладкой класса $C^{2}$ границей.

Изучается асимптотическое поведение при $t \rightarrow \infty$ решений уравнения (1), удовлетворяющих условию:

$$
\frac{\partial u}{\partial \nu}=0, \quad x \in \partial \Omega, \quad t>0
$$

где $\nu$ - вектор единичной внешней нормали к $\partial \Omega$.

Предполагается, что $\sigma$ - положительная константа, $a(x)$ - неотрицательная ограниченная функция в $\bar{\Omega}$ со средним значением

$$
\bar{a}=(\operatorname{mes} \Omega)^{-1} \int_{\Omega} a(x) d x>0 .
$$

Будем использовать обозначения $\Pi_{a, b}=\{(x, t) \mid x \in \Omega, a<t<b\}, S_{a, b}=$ $\{(x, t) \mid x \in \partial \Omega, a<t<b\}, \Pi_{a}=\Pi_{a, \infty}, S_{a}=S_{a, \infty}$.

Под решением в $\Pi_{0}$ уравнения (1), удовлетворяющим условию (2), понимается обобшенное решение в стандартном определении, т.е. функция $u(x, t)$, принадлежащая $W_{2}^{1,1}\left(\Pi_{a, b}\right) \cap L_{\infty}\left(\Pi_{a, b}\right)$ при любых $0<a<b$ и удовлетворяющая интегральному тождеству

$$
\int_{\Pi_{a, b}} u_{t} \psi d x d t+\sum_{i=1}^{n} \int_{\Pi_{a, b}} \frac{\partial u}{\partial x_{i}} \frac{\partial \psi}{\partial x_{i}} d x d t+\int_{\Pi_{a, b}} a(x)|u|^{\sigma} u \psi d x d t=0
$$

для любой функции $\psi(x, t) \in W_{2}^{1,0}\left(\Pi_{a, b}\right)$.

Работа вьполнена при поддержке фонда INTAS (грант № 03-51-5007) и Программы поддержки ведущих научных школ РФ (грант № НШ-1464.2003.1).

(C) и. В. ФилимоновА 2004 
Из теории линейных параболических уравнений, изложенной, например, в [1], следует, что обобщенные решения уравнения (1), удовлетворяюшие (2), непрерывны в $\Pi_{0}$ вплоть до $S_{0}$, принадлежат $W_{2}^{2,1}\left(\Pi_{a, b}\right)$ и удовлетворяют уравнению (1) почти всюду.

Исследованию асимптотического поведения решений уравнения (1), удовлетворяюших условию (2), посвящены работы [2]-[4] и др.

В работе [3] доказано, что все решения уравнения (1), удовлетворяюшие условию $(2)$, стремятся к нулю при $t \rightarrow \infty$, и найден первьй член асимптотики решения при $t \rightarrow \infty$. А именно доказано, что для любого решения сушествует равномерный по $\Omega$ предел при $t \rightarrow \infty$ :

$$
t^{1 / \sigma} u(x, t) \underset{\Omega}{\rightrightarrows} \Lambda \in\left\{-c_{0}, 0, c_{0}\right\}, \quad c_{0}=(\sigma \bar{a})^{-1 / \sigma} .
$$

В работе [4] установлено, что если $a(x) \equiv a_{0}=$ const $>0$, то любое решение уравнения (1), удовлетворяющее граничному условию (2), имеет вид

$$
u(x, t)=C(t+\tau)^{-1 / \sigma}+o\left(e^{-\alpha t}\right), \quad t \rightarrow \infty,
$$

где либо $C=0$, либо $|C|=\left(\sigma a_{0}\right)^{-1 / \sigma} ;$ константа $\tau$ зависит от $u$, а константа $\alpha>0$ от $u$ не зависит. Более того, $C=0$ тогда и только тогда, когда решение обрашается в нуль при сколь угодно больших $t$.

В настоящей работе доказывается, что:

1) осциллирующее, т.е. обращающееся в нуль при сколь угодно больших $t$, решение уравнения (1), для которого выполнено условие (2), экспоненциально убьвает при $t \rightarrow \infty$ (отметим, что при любом $\alpha$ уравнение (1) имеет решение, удовлетворяюшее условию (2) и убываюшее быстрее, чем $\exp (\alpha t))$;

2 для любых двух положительных при $t \rightarrow \infty$ решений $u(x, t)$ и $v(x, t)$ уравнения (1), удовлетворяющих условию (2), существует такая константа $\tau$, что $u(x, t)-$ $v(x, t+\tau)=o\left(e^{-\alpha t}\right)$, где константа $\alpha>0$ не зависит от $u, v$;

$3)$ для любого положительного решения $u(x, t)$ уравнения (1), удовлетворяющего условию (2), можно при любом $N$ подобрать непрерывные в $\bar{\Omega}$ функции $\varphi_{k i}(x)$, $k=0, \ldots, N, i=0, \ldots, k$, такие, что

$$
u(x, t)=\sum_{k=0}^{N} t^{-1 / \sigma-k} \sum_{i=0}^{k} \varphi_{k i}(x) \ln ^{i} t+o\left(t^{-1 / \sigma-N}\right), \quad t \rightarrow \infty,
$$

причем $\varphi_{00}=(\sigma \bar{a})^{-1 / \sigma}$.

\section{§ 2. Предварительные сведения}

Для доказательства теорем об асимптотическом поведении решений уравнения (1), удовлетворяющих условию (2), используется ряд результатов об асимптотическом поведении решений линейных уравнений.

Сформулируем их.

Пусть $u(x, t)$ - решение уравнения

$$
u_{t}=\Delta u+a(x, t) u+f(x, t), \quad x \in \Omega, \quad t \in \mathbb{R},
$$


такое, что

$$
\left.\frac{\partial u}{\partial \nu}\right|_{\partial \Omega \times \mathbb{R}}=0 .
$$

Доказательства следующих двух утверждений изложены в работе [5].

ПРЕДЛОЖЕНИЕ 1. Пусть

$$
\begin{array}{ll}
\left(\int_{\Omega} a^{2}(x, t) d x\right)^{1 / 2}=O\left(t^{-1}\right), & t \rightarrow \infty, \\
\int_{\Omega} a(x, t) d x=a_{0} t^{-1}+o\left(t^{-1-\varepsilon}\right), & t \rightarrow \infty,
\end{array}
$$

где $a_{0}=$ const, $\varepsilon>0$. Пусть функиия $f(x, t)$ равна нулю при $t<1$ и такова, что при некотором $\sigma>1 / 2-a_{0}$ выполнено

$$
\int_{1}^{\infty} \int_{\Omega}|f(x, t)|^{2} t^{2 \sigma} d x d t<\infty
$$

Пусть константа $\delta>0$ такова, что в полосе $|\operatorname{Re} \lambda|<\delta$ нет точек спектра задачи

$$
\Delta u+\lambda u=0, \quad x \in \Omega,\left.\quad \frac{\partial u}{\partial \nu}\right|_{\partial \Omega}=0
$$

отличных от $\lambda=0$.

Тогда решение $и(x, t)$ задачи $(3),(4)$ такое, что

$$
\int_{1}^{\infty} \int_{\Omega}\left(|u|^{2}+|\Delta u|^{2}+\left|u_{t}\right|^{2}\right) e^{-2 \delta t} d x d t<\infty
$$

имеет вид

$$
u(x, t)=c t^{a_{0}}+o\left(t^{a_{0}}\right), \quad t \rightarrow \infty,
$$

əдe $c=$ const.

ПРЕДЛОЖЕНИЕ 2. Пусть выполнены условия предложсения 1. Найдется $t_{0}(\sigma)$ такое, что существует решение задачи $(3),(4)$, удовлетворяющее условию

$$
\int_{t_{0}}^{\infty} \int_{\Omega}\left[|u|^{2} t^{2 \sigma-2}+\left(|\Delta u|^{2}+\left|u_{t}\right|^{2}\right) t^{2 \sigma-2}\right] d x d t<\infty .
$$

Будет также использоваться следующее

ПРЕДЛОЖЕНИЕ 3. Каково бъ ни бъло $\alpha, 0<\alpha<\lambda_{1}$, әде $\lambda_{1}-$ первое ненулевое собственное значение задачи (5), найдется такое $\nu>0$, что если $|a(x, t)|<\nu, a(x, t)=0$ nри $t<1$ u $f(x, t)$ - финитная по $t$ функиия, то существует решение уравнения (3), удовлетворяющее условию (4), такое, чmo

$$
u(x, t)= \begin{cases}o\left(e^{-\alpha t}\right), & t \rightarrow+\infty \\ C+o\left(e^{\alpha t}\right), & t \rightarrow-\infty\end{cases}
$$


ДокАЗАТЕЛЬСтво. Введем функциональные пространства $L_{2, q}$ и $W_{2, q}^{2,1}$ с нормами

$$
\begin{aligned}
\|f\|_{L_{2, q}}^{2} & =\int_{\mathbb{R}} \int_{\Omega} f^{2} e^{2 q t} d x d t<\infty, \\
\|u\|_{W_{2, q}^{2,1}}^{2} & =\int_{\mathbb{R}} \int_{\Omega}\left(u_{t}^{2}+|\Delta u|^{2}+u^{2}\right) e^{2 q t} d x d t<\infty
\end{aligned}
$$

соответственно.

Пусть $\alpha$-произвольная положительная константа, меньшая чем $\lambda_{1}-$ первое ненулевое собственное значение задачи (5). Введем оператор $Q: L_{2, \alpha} \rightarrow W_{2, \alpha}^{2,1}$, переводяший $f(x, t) \in L_{2, \alpha}$ в решение $u(x, t)$ уравнения $u_{t}=\Delta u+f(x, t)$ в $\Omega \times \mathbb{R}$, удовлетворяющее условию (4) и принадлежашее $W_{2, \alpha}^{2,1}$. Такое решение сушествует, единственно и может быть построено методом преобразования Фурье по $t$. Действительно, рассмотрим задачу с вешественньм параметром $\xi$ :

$$
i \xi \widehat{w}=(\Delta+\alpha) \widehat{w}+\widehat{f}_{1}, \quad x \in \Omega,\left.\quad \frac{\partial \widehat{w}}{\partial \nu}\right|_{\partial \Omega}=0,
$$

где $f_{1}=f \exp (\alpha t)$, а $\widehat{f}_{1}$ - образ функции $f_{1}$ при преобразовании Фурье по $t$.

Так как на прямой $\operatorname{Re} \lambda=\alpha$ нет точек спектра задачи $(5)$, то при любом $\xi \in \mathbb{R}$ существует единственное решение $\widehat{w}(\xi)=R(\alpha-i \xi) \widehat{f}_{1}$ задачи $(7)$ из $W_{2}^{2}(\Omega)$, где $R(\lambda)$ - резольвента задачи (5). В [6] показано, что

$$
\|R(\alpha-i \xi)\|_{L_{2}(\Omega) \rightarrow L_{2}(\Omega)} \leqslant C(1+|\xi|)^{-1},
$$

поэтому $\widehat{w}(\xi), \xi \widehat{w}(\xi) \in L_{2,0}$ и обратное преобразование Фурье по $\xi$ от $\widehat{w}(\xi)$ сушествует и удовлетворяет уравнению $u_{t}=\Delta u+f(x, t)$ и условию (4).

Единственность решения, принадлежашего $W_{2, \alpha}^{2,1}$, следует из того, что к таким решениям, умноженным на $\exp (\alpha t)$, применимо преобразование Фурье, а однородная задача (7) при любом значении параметра $\xi \in \mathbb{R}$ имеет только нулевое решение.

Таким образом, оператор $Q$ определен. Из оценки нормы оператора $R(\alpha-i \xi)$ в $L_{2}(\Omega)$ и равенства Парсеваля следует, что оператор $Q$ ограничен как оператор из $L_{2, \alpha}$ в $L_{2, \alpha}$.

Пусть $g \in L_{2, \alpha}$. Если $g+a(x, t) Q g+f(x, t)=0$, то функция $Q g$ будет решением задачи $(3),(4)$. Применяя теорему об обратимости оператора, мало отличающегося по норме от единичного, находим, что при $\sup |a(x, t)|<1 /(2\|Q\|) \doteq \nu$ решением $u_{1}(x, t)$ уравнения (3), удовлетворяюшим условию (4), является функция $Q g$.

Так как $f(x, t)$ финитна по $t$, то при достаточно больших $N$ функция $u=u_{1} e^{\alpha t}$ является в $\Pi_{N-2, N+2}$ решением уравнения $u_{t}=\Delta u+\alpha u+a(x, t) u$, удовлетворяюшим условию (4). Из классической оценки Нэша [1] следует, что

$$
\max _{(x, t) \in \Pi_{N-1, N+1}} u_{1}(x, t) e^{\alpha t} \leqslant C\left(\int_{\Pi_{N-2, N+2}} u_{1}^{2} e^{2 \alpha t} d x d t\right)^{1 / 2}
$$

при этом константа $C=C(\alpha, \nu)$ не зависит от $N$. Так как $u_{1}(x, t) \in L_{2, \alpha}$, то при $N \rightarrow+\infty$ получим, что $u_{1}(x, t)=o\left(e^{-\alpha t}\right), t \rightarrow+\infty$. 
Пусть $\zeta(t)=1$ при $t<0$ и $\zeta(t)=0$ при $t>1$. Тогда $u_{2}=u_{1} \zeta(t)-$ решение задачи вида $(3),(4)$ с $a(x, t) \equiv 0$ и некоторой финитной по $t$ функцией $f(x, t)$.

Так как корневое подпространство, отвечающее $\lambda=0$, одномерно и порождено собственной функцией, равной константе, то для резольвенты $R(\lambda)$ задачи (5) в некоторой окрестности $\lambda=0$ имеет место представление

$$
R(\lambda) f=\lambda^{-1} \bar{f}+\Gamma(\lambda) f
$$

где $\bar{f}$ - среднее значение функции $f$ в области $\Omega, \Gamma(\lambda)$ - аналитическая операторнозначная функция [7]. Так как в полосе $|\operatorname{Re} \lambda|<\alpha+\varepsilon, 0<\varepsilon<\lambda_{1}-\alpha$, задача (5) имеет только нулевое собственное значение, то в этой полосе при $\lambda \neq 0$ резольвента $R(\lambda)$ аналитична, если же $|\lambda|>\mu$, где $\mu$ произвольно мало, то она равномерно ограничена в этой полосе. Следовательно, представление (8) имеет место во всей полосе $|\operatorname{Re} \lambda|<\alpha+\varepsilon$ c аналитической и ограниченной функцией $\Gamma(\lambda)$.

Из результатов Агмона, Ниренберга [8] и из представления (8) следует, что $u_{2}(x, t)$ при $t \rightarrow-\infty$ имеет вид $u_{2}(x, t)=C+v$, где $C=$ const, $v \in W_{2,-\alpha}^{2,1}$.

Аналогично предыдушему, рассматривая уравнение для $v$ и используя оценку Нэша, получаем, что $v=o\left(e^{\alpha t}\right)$ при $t \rightarrow-\infty$.

Таким образом, $u_{1}(x, t)$ имеет вид (6) и предложение 3 доказано.

Прежде чем перейти к последовательному изложению результатов, отметим следующее

УТВЕРЖДЕНИЕ 1. Для любого решения $и(x, t)$ уравнения (1), удовлетворяющего граничному условию (2), выполнена оценка

$$
|u(x, t)|<k t^{-1 / \sigma}, \quad t>0,
$$

əде константа $k$ зависит от и.

ДокАЗАТЕльство. Действительно, так как $\bar{a}>0$, то при достаточно большом $k \eta=k / \sigma-\bar{a} k^{\sigma+1}<0$. Определим непрерывную в $\bar{\Omega}$ функцию $\varphi(x)$ как решение задачи

$$
\Delta \varphi=\frac{1}{\sigma} k-a(x) k^{\sigma+1}-\eta, \quad x \in \Omega,\left.\quad \frac{\partial \varphi}{\partial \nu}\right|_{\partial \Omega}=0 .
$$

Эта задача разрешима в силу выбора $\eta$, и у нее сушествует положительное в $\bar{\Omega}$ решение $\varphi(x)$. Функция $w_{\text {sup }}(x, t)=k t^{-1 / \sigma}+\varphi(x) t^{-2-1 / \sigma}>0$ является при $t>t_{1}$ суперрешением уравнения (1), поскольку

$$
\Delta w_{\text {sup }}-a(x) w_{\text {sup }}^{\sigma+1}-\left(w_{\text {sup }}\right)_{t}=\eta t^{-1-1 / \sigma}+O\left(t^{-2-1 / \sigma}\right)<0
$$

для достаточно больших $t>t_{1}$. Следовательно, функция $w_{\mathrm{sup}}\left(x, t+t_{1}\right)$ является при $t>0$ суперрешением уравнения (1), удовлетворяюшим условию (2). Так как $a(x) \geqslant 0$, то при любом $c>1$ функция $c w_{\text {sup }}\left(x, t+t_{1}\right)$ - также суперрешение уравнения (1). Отсюда следует, что для любого решения $u(x, t)$ можно так подобрать $c_{u}>1$, что $u(x, t) \leqslant c_{u} w_{\sup }\left(x, t+t_{1}\right)$ при $t=0, x \in \Omega$. Из принципа максимума и вида $w_{\mathrm{sup}}(x, t)$ заключаем, что для любого решения имеет место оценка $(9)$. 


\section{§ 3. Экспоненциальное убывание осциллирующих решений}

ТЕОРема 1. Если решение и $(x, t)$ в $\Pi_{0}$ уравнения (1), удовлетворяющее условию (2), обращается в нуль в точках $\left(x_{N}, t_{N}\right), t_{N} \rightarrow+\infty$, то

$$
|u(x, t)| \leqslant c \exp (-\alpha t)
$$

где с и $\alpha$ - положительные константы, причем $\alpha$ не зависит от и.

ДокАЗАТЕЛЬСТво. Пусть $\zeta(t)$ - гладкая класса $C^{1}$ функция, $\zeta(t)=0$ при $t<t_{0}$ и $\zeta(t)=1$ при $t>t_{0}+1$, константа $t_{0}>1$ будет выбрана позже.

Функция

$$
u_{1}(x, t)= \begin{cases}u(x, t) \zeta(t), & t>0 \\ 0, & t \leqslant 0\end{cases}
$$

удовлетворяет в $\Omega \times \mathbb{R}$ уравнению

$$
\left(u_{1}\right)_{t}=\Delta u_{1}-Q(x, t) u_{1}+F(x, t)
$$

и условию

$$
\left.\frac{\partial u}{\partial \nu}\right|_{\partial \Omega \times \mathbb{R}}=0 .
$$

Здесь $F(x, t)=u \zeta_{t}, Q(x, t)=a(x)|u(x, t)|^{\sigma} \zeta(t)$. Функции $F(x, t), Q(x, t)$ по определению полагаются равными нулю при $t \leqslant t_{0}$.

Так как $u(x, t)$ стремится к нулю при $t \rightarrow \infty[3]$, то константу $t_{0}$ можно выбрать так, чтобы $|Q|<\nu$ при $t>t_{0}$, где $\nu$ определено в предложении 3.

Следовательно, применив предложение 3 , найдем, что уравнение (10) имеет решение $u_{2}(x, t)$ такое, что

$$
u_{2}(x, t)= \begin{cases}o\left(e^{-\alpha t}\right), & t \rightarrow+\infty \\ C+o\left(e^{\alpha t}\right), & t \rightarrow-\infty\end{cases}
$$

где $C$ и $\alpha$ - константы, причем $\alpha>0$.

Функция $w=u_{1}-u_{2}$ удовлетворяет уравнению $w_{t}=\Delta w-Q(x, t) w$ и $w=C+$ $o\left(e^{\alpha t}\right)$ при $t \rightarrow-\infty$. Если $C>0$, то из принципа максимума следует, что $w$ положительна.

Уравнение $w_{t}=\Delta w-Q(x, t) w$ имеет положительное субрешение $w_{\mathrm{sub}}(t)$, зависящее только от $t$ и убываюшее при $t \rightarrow \infty$ степенным образом. Действительно, так как из (9) имеем оценку $|Q|=|a(x)||u(x, t)|^{\sigma} \zeta(t) \leqslant c_{2} k^{\sigma} t^{-1}$, где $c_{2}$ - такая постоянная, что $a(x)<c_{2}$, то

$$
\Delta w_{\mathrm{sub}}-Q(x, t) w_{\mathrm{sub}}-\left(w_{\mathrm{sub}}\right)_{t} \geqslant-\left(w_{\mathrm{sub}}\right)_{t}-c_{2} k_{u}^{\sigma} t^{-1} w_{\mathrm{sub}}
$$

Следовательно, в качестве искомого субрешения можно взять любое решение уравнения $\left(w_{\mathrm{sub}}\right)_{t}+c_{2} k_{u}^{\sigma} t^{-1} w_{\mathrm{sub}}=0$, например $w_{\mathrm{sub}}=c t^{-c_{2} k_{0}^{\sigma} \text { с произвольной кон- }}$ стантой $c$.

Определим теперь константу $c$, входящую в $w_{\mathrm{sub}}$, так, чтобы при $t=1, x \in \Omega$ было выполнено неравенство $w \geqslant w_{\text {sub }}$. По принципу максимума такая же оценка 
будет верна и при $t>1, x \in \Omega$. Это противоречит тому, что $w\left(x_{N}, t_{N}\right)=o\left(e^{-\alpha t_{N}}\right)$, где $\left(x_{N}, t_{N}\right)$ - такая последовательность, что $u_{1}\left(x_{N}, t_{N}\right)=0$ и $t_{N} \rightarrow \infty$.

Таким образом, допущение $C>0$ неверно; аналогично, неверно и допущение $C<0$. Следовательно, $C=0$. Поэтому функция $w(x, t)$, являюшаяся решением уравнения (10) в случае $F(x, t)=0$, стремится к нулю как при $t \rightarrow+\infty$, так и при $t \rightarrow-\infty$. Из принципа максимума $w(x, t) \equiv 0$ и, следовательно, при больших значениях $t$ имеем $u(x, t)=u_{1}(x, t)=u_{2}(x, t)=o\left(e^{-\alpha t}\right)$, что и требовалось доказать.

В случае $a(x)=$ const теорема 1 совпадает с леммой 2 работы [4].

Переходим к изучению решений, которые сохраняют знак при $t>T$. Не нарушая общности, можно ограничиться рассмотрением положительных решений.

\section{§4. Асимптотическая эквивалентность положительных решений}

Сначала покажем, что в формуле

$$
u_{N}(x, t)=\sum_{k=0}^{N} t^{-1 / \sigma-k} \sum_{i=0}^{k} \varphi_{k i}(x) \ln ^{i} t
$$

можно так подобрать непрерывные в $\bar{\Omega}$ функции $\varphi_{k i}(x), \varphi_{00}(x)>0$, что

$$
f_{N}(x, t) \equiv\left(u_{N}\right)_{t}-\Delta u_{N}+a(x)\left|u_{N}\right|^{\sigma} u_{N}=o\left(t^{-1 / \sigma-N}\right), \quad t \rightarrow \infty .
$$

Вычислим все члены, входящие в левую часть (12). Ясно, что

$$
\left(u_{N}\right)_{t}(x, t)=\sum_{k=0}^{N} t^{-1 / \sigma-k-1} \sum_{i=0}^{k} \varphi_{k i}(x)\left(i \ln ^{i-1} t-\left(k+\frac{1}{\sigma}\right) \ln ^{i} t\right) .
$$

Для вычисления $\left(u_{N}\right)^{\sigma+1}$ используем разложение Тейлора

$$
(c+h)^{\sigma+1}=c^{\sigma+1}+\sum_{l=1}^{N}\left(\begin{array}{c}
\sigma+1 \\
l
\end{array}\right) c^{\sigma+1-l} h^{l}+o\left(h^{N}\right), \quad h \rightarrow 0,
$$

где $c>0,\left(\begin{array}{c}\sigma+1 \\ l\end{array}\right)=\frac{(\sigma+1) \sigma \cdots(\sigma-l+2)}{l !}-$ биномиальные коэффициенты.

Для удобства введем обозначение

$$
\widetilde{u}_{N}(x, t)=\sum_{k=1}^{N} t^{-k} \sum_{i=0}^{k} \varphi_{k i}(x) \ln ^{i} t .
$$

Проведем теперь вычисление $\left(u_{N}\right)^{\sigma+1}$ :

$$
\begin{aligned}
\left(u_{N}\right)^{\sigma+1} & =\left[t^{-1 / \sigma}\left(\varphi_{00}+\widetilde{u}_{N}\right)\right]^{\sigma+1} \\
& =t^{-1 / \sigma-1}\left[\varphi_{00}^{\sigma+1}+\sum_{l=1}^{N}\left(\begin{array}{c}
\sigma+1 \\
l
\end{array}\right) \varphi_{00}^{\sigma+1-l}\left(\widetilde{u}_{N}\right)^{l}+o\left(\widetilde{u}_{N}^{N}\right)\right] .
\end{aligned}
$$


Заметим, что $t^{N-1} \widetilde{u}_{N}^{N}$ стремится к нулю при $t \rightarrow \infty$ и, следовательно,

$$
\begin{aligned}
\left(u_{N}\right)^{\sigma+1}= & t^{-1 / \sigma-1}\left[\varphi_{00}^{\sigma+1}+(\sigma+1) \varphi_{00}^{\sigma} \widetilde{u}_{N}\right. \\
& \left.+\sum_{l=2}^{N-1}\left(\begin{array}{c}
\sigma+1 \\
l
\end{array}\right) \varphi_{00}^{\sigma+1-l}\left(\widetilde{u}_{N}\right)^{l}+o\left(t^{1-N}\right)\right]
\end{aligned}
$$

при $t \rightarrow \infty$. Отметим, что при $l \geqslant 2$

$$
\left(\widetilde{u}_{N}\right)^{l}=\sum_{k=l}^{N} t^{-k} \sum_{i=0}^{k} \psi_{l k i}(x) \ln ^{i} t+o\left(t^{-N}\right), \quad t \rightarrow \infty,
$$

где функции $\psi_{l k i}(x)$ - линейные комбинации произведений нескольких функций $\varphi_{s j}$, $1 \leqslant s \leqslant k-l+1<k, 0 \leqslant j \leqslant k$. Следовательно, при $t \rightarrow \infty$

$$
\begin{aligned}
u_{N}^{\sigma+1}= & t^{-1 / \sigma-1} \varphi_{00}^{\sigma+1}+\varphi_{00}^{\sigma}\left[\sum_{k=1}^{N} t^{-1 / \sigma-k-1} \sum_{i=0}^{k}\left((\sigma+1) \varphi_{k i}+\Phi_{k i}\right) \ln ^{i} t\right] \\
& +o\left(t^{-1 / \sigma-N}\right),
\end{aligned}
$$

где

$$
\Phi_{k i}(x)=\sum_{l=2}^{N-1}\left(\begin{array}{c}
\sigma+1 \\
l
\end{array}\right) \varphi_{00}^{-l+1} \psi_{l k i} .
$$

Подставив (13), (14) в (12), получим, что коэффициент при $t^{-1 / \sigma-k-1} \ln ^{i} t$ в случае $i=k+1$ при произвольном $k,-1 \leqslant k \leqslant N-1$, равен $\Delta \varphi_{k+1, k+1}$; если $0<k \leqslant N-1,0 \leqslant i \leqslant k$, этот коэффициент равен

$$
(1+i) \varphi_{k, i+1}-\left(k+\frac{1}{\sigma}\right) \varphi_{k, i}-\Delta \varphi_{k+1, i}+a(x) \varphi_{00}^{\sigma}\left[(\sigma+1) \varphi_{k, i}+\Phi_{k, i}\right],
$$

а при $k=i=0$ он оказьвается следующим:

$$
\frac{1}{\sigma} \varphi_{00}-\Delta \varphi_{10}+a(x) \varphi_{00}^{\sigma+1}
$$

Приравнивая эти коэффициенты к нулю, получаем уравнения для $\varphi_{k+1, i}, 0 \leqslant i \leqslant$ $k+1$, с нулевым граничным условием Неймана:

- при $i=k+1$ и $-1 \leqslant k \leqslant N-1$

$$
\Delta \varphi_{k+1, k+1}=0,
$$

следовательно, $\varphi_{k+1, k+1}=$ const, константу $\varphi_{00}$ далее обозначаем $c_{0}$;

- при $k=i=0$

$$
\Delta \varphi_{10}=-\frac{1}{\sigma} c_{0}+a(x) c_{0}^{\sigma+1}
$$

- при $1 \leqslant k \leqslant N-1$ и $0 \leqslant i \leqslant k$

$$
\begin{aligned}
\Delta \varphi_{k+1, i}= & -\left(k+\frac{1}{\sigma}\right) \varphi_{k, i}+(1+i) \varphi_{k, i+1} \\
& +a(x) c_{0}^{\sigma}\left((\sigma+1) \varphi_{k, i}+\Phi_{k i}\right) .
\end{aligned}
$$


Поскольку $\Phi_{k i}(x)=0$ при $k=1$, то уравнения для $\varphi_{20}, \varphi_{21}$ таковы:

$$
\begin{aligned}
& \Delta \varphi_{20}=-\left(\frac{1}{\sigma}+1\right) \varphi_{10}+\varphi_{11}+a(x)(\sigma+1) c_{0}^{\sigma} \varphi_{10} \\
& \Delta \varphi_{21}=-\left(\frac{1}{\sigma}+1\right) \varphi_{11}+a(x)(\sigma+1) c_{0}^{\sigma} \varphi_{11} .
\end{aligned}
$$

Покажем, что из этой системы уравнений можно последовательно определить все функции $\varphi_{k i}(x), i \leqslant k$. Пусть при фиксированном $k$ выполнены условия разрешимости для уравнений, определяющих $\varphi_{k i}$, т.е. среднее значение их правых частей равно нулю. Тогда $\varphi_{k i}$ можно записать в виде $\varphi_{k i}=\psi_{k i}+c_{k i}$, где $\bar{\psi}_{k i}=0$, а $c_{k i}$ - пока еше не определенные постоянные. Константы $c_{k i}$ надо подобрать так, чтобы были выполнены условия разрешимости для уравнений, определяющих $\varphi_{k+1, i}$.

Проверим, что такой выбор возможен при любом $k$.

При $k=0$ из условия разрешимости уравнения для $\varphi_{10}$

$$
\int_{\Omega}\left(-\frac{1}{\sigma} c_{0}+a(x) c_{0}^{\sigma+1}\right) d x=0
$$

находим

$$
c_{0}=(\sigma \bar{a})^{-1 / \sigma} .
$$

При $k>0$ уравнения для $c_{k i}$ будут получаться линейными, причем если при фиксированном $k$ условия разрешимости выписывать последовательно, в убывающем порядке от $i=k$ до $i=0$, то в выражении для $\Delta \varphi_{k+1, i}$ останется не определенной только константа $c_{k i}$, коэффициент при которой равен

$$
\overline{a(x)}(\sigma+1) c_{0}^{\sigma}-k-\frac{1}{\sigma} \equiv 1-k .
$$

Поэтому $c_{k i}$ при $k>1$ находятся однозначно. Осталось подобрать $c_{10}, c_{11}$. Так как $\varphi_{11}=c_{11}=$ const, то условие разрешимости уравнения, определяюшего $\varphi_{21}$ :

$$
c_{11} \int_{\Omega}\left(-\left(\frac{1}{\sigma}+1\right)+a(x)(\sigma+1) c_{0}^{\sigma}\right) d x=0,
$$

вьполнено в силу (15). Из условия разрешимости уравнения для $\varphi_{20}$

$$
c_{11} \operatorname{mes} \Omega=\int_{\Omega}(\sigma+1) \varphi_{10}(x)\left(\frac{1}{\sigma}-a(x) c_{0}^{\sigma}\right) d x
$$

находится $c_{11}$. Константу $c_{10}$ можно считать произвольной. Тем самым, требуемый выбор констант $c_{k i}$ возможен. Итак, при фиксированном произволе в выборе $\varphi_{10}$ все коэффициенты $\varphi_{k i}$ при $k \neq N$ определяются однозначно, а при $k=N$ с точностью до произвольной константы.

Отметим следуюшее

УТВЕРЖДЕНИЕ 2. Константа с 11 равна нулю тогда и только тогда, когда $a(x) \equiv$ const. 
ДокАЗАТЕЛЬство. Действительно, условие (15) можно переписать следующим образом:

$$
c_{11} \operatorname{mes}(\Omega)=-\int_{\Omega} \frac{\sigma+1}{c_{0}} \varphi_{10} \Delta \varphi_{10} d x=0 \quad \Longleftrightarrow \nabla \varphi_{10}=0 \quad \Longleftrightarrow \quad \varphi_{10}=\text { const. }
$$

Из уравнения для функции $\varphi_{10}$ следует, что $\Delta \varphi_{10}=0$ тогда и только тогда, когда $-\frac{1}{\sigma} c_{0}+a(x) c_{0}^{\sigma+1}=0$, т.е. $a(x) \equiv\left(c_{0}^{\sigma} \sigma\right)^{-1}$.

ЛЕмма 1. Все положительнье при $t>T$ решения уравнения (1), удовлетворяющие условию (2), имеют вид

$$
u(t, x)=c_{0} t^{-1 / \sigma}+c_{11} t^{-(\sigma+1) / \sigma} \ln t+o\left(t^{-(\sigma+1) / \sigma} \ln t\right), \quad t \rightarrow+\infty,
$$

где с $c_{0}, c_{11}$ определены в (15), (16).

ДоказАТЕЛЬство. Рассмотрим следуюшую систему для определения функций $\varphi_{10}(x), \varphi_{20}(x), \varphi_{21}(x)$, удовлетворяюших условию $(2)$ :

$$
\begin{aligned}
& \Delta \varphi_{10}=-\frac{1}{\sigma} c_{0}+a(x) c_{0}^{\sigma+1} \\
& \Delta \varphi_{20}=\varepsilon-\left(\frac{1}{\sigma}+1\right) \varphi_{10}+c_{11}(\varepsilon)+a(x)(\sigma+1) c_{0}^{\sigma} \varphi_{10} \\
& \Delta \varphi_{21}=-\left(\frac{1}{\sigma}+1\right) c_{11}(\varepsilon)+a(x)(\sigma+1) c_{0}^{\sigma} c_{11}(\varepsilon) .
\end{aligned}
$$

В силу (15) условия разрешимости уравнений для $\varphi_{10}(x), \varphi_{21}(x)$ выполнены. Из условия разрешимости уравнения для $\varphi_{20}(x)$ следует, что $c_{11}(\varepsilon)=c_{11}-\varepsilon$. Зафиксируем $\varepsilon>0$ и рассмотрим функцию

$$
\begin{aligned}
w(x, t)= & c_{0} t^{-1 / \sigma}+c_{11}(\varepsilon) t^{-(1+1 / \sigma)} \ln t+\varphi_{10}(x) t^{-(1+1 / \sigma)} \\
& +\varphi_{21}(x) t^{-(2+1 / \sigma)} \ln t+\varphi_{20}(x) t^{-(2+1 / \sigma)}
\end{aligned}
$$

где константы, с точностью до которых определены функции $\varphi_{10}(x), \varphi_{20}(x), \varphi_{21}(x)$, произвольно зафиксированы. Так как

$$
\Delta w-a(x)|w|^{\sigma} w-w_{t}=\varepsilon t^{-2-1 / \sigma}+O\left(t^{-3-1 / \sigma} \ln ^{2} t\right),
$$

то при достаточно большом $t>t_{0}$ выполнено

$$
\Delta w-a(x)|w|^{\sigma} w-w_{t}>0
$$

Выберем $\tau>0$ так, что $w\left(x, t_{0}+\tau\right)<u\left(x, t_{0}\right)$; это возможно, поскольку $w(x, t) \rightarrow 0$ при $t \rightarrow \infty$, a $u\left(x, t_{0}\right)$ положительна. Из принципа максимума получаем, что $u(x, t)>w(x, t+\tau)$ при $t>t_{0}$. Учитывая явный вид $w(x, t)(16)$, можно переписать это неравенство:

$$
u(x, t)>c_{0} t^{-1 / \sigma}+c_{11}(\varepsilon) t^{-(1+1 / \sigma)} \ln t+O\left(t^{-(1+1 / \sigma)}\right) .
$$


Аналогично, если $\varepsilon<0$, то при достаточно большом $t>t_{0}$ функция $w$ положительна и удовлетворяет неравенству

$$
\Delta w-a(x)|w|^{\sigma} w-w_{t}<0
$$

Поскольку $u(x, t) \rightarrow 0$ при $t \rightarrow \infty$, то существует такое $\tau>0$, что $u\left(x, t_{0}+\tau\right)<$ $w\left(x, t_{0}\right)$, а значит, при $t>t_{0}+\tau$ из принципа максимума следует, что $u(x, t)<$ $w(x, t-\tau)$. Учитывая (17), последнее неравенство можно записать в виде:

$$
u(x, t)<c_{0} t^{-1 / \sigma}+c_{11}(\varepsilon) t^{-(1+1 / \sigma)} \ln t+O\left(t^{-(1+1 / \sigma)}\right) .
$$

Докажем, что

$$
u(t, x) \sim c_{0} t^{-1 / \sigma}+c_{11} t^{-(\sigma+1) / \sigma} \ln t .
$$

Из неравенств $(18),(19)$ для $\varepsilon= \pm \varepsilon_{0}$ при $t>t_{1}$ получаем

$$
\begin{aligned}
& \left(c_{11}\left(\varepsilon_{0}\right)-c_{11}\right) t^{-(1+1 / \sigma)} \ln t+O\left(t^{-(1+1 / \sigma)}\right)<u(x, t)-c_{0} t^{-1 / \sigma}-c_{11} t^{-(\sigma+1) / \sigma} \ln t \\
& \quad<\left(c_{11}\left(-\varepsilon_{0}\right)-c_{11}\right) t^{-(1+1 / \sigma)} \ln t+O\left(t^{-(1+1 / \sigma)}\right) .
\end{aligned}
$$

Разделив это неравенство на $t^{-(\sigma+1) / \sigma} \ln t$ и учитывая, что $c_{11}(\varepsilon)=c_{11}-\varepsilon$, получим

$$
\left|\frac{u(x, t)-c_{0} t^{-1 / \sigma}-c_{11} t^{-(\sigma+1) / \sigma} \ln t}{t^{-(\sigma+1) / \sigma} \ln t}\right|<\varepsilon_{0}+O\left((\ln t)^{-1}\right) .
$$

Увеличив, если надо, $t_{1}$, найдем, что при $t>t_{1}$ имеет место

$$
\left|\frac{u(x, t)-c_{0} t^{-1 / \sigma}-c_{11} t^{-(\sigma+1) / \sigma} \ln t}{t^{-(\sigma+1) / \sigma} \ln t}\right|<2 \varepsilon_{0},
$$

тем самым лемма доказана.

\section{§5. Асимптотическое разложение положительных решений}

ТЕОРема 2. Положительное решение уравнения (1), удовлетворяющее условию (2), имеет вид

$$
u(x, t)=u_{N}(x, t)+o\left(t^{1+3 / 4-1 / \sigma-N}\right), \quad t \rightarrow \infty
$$

где $u_{N}(x, t)$ определено в (11) с константой $c_{10}$, зависящей от $u(x, t)$.

ДоказАТЕльство. Делая в уравнении (1) замену $u=v+u_{N}$, где $u_{N}$ определено в (11) с произвольно выбранной константой $c_{10}$, получим

$$
v_{t}=\Delta v-a(x) \frac{\left(v+u_{N}\right)^{\sigma+1}-\left(u_{N}\right)^{\sigma+1}}{v} v+f_{N},
$$

$f_{N}$ определено в (12). 
Учитьвая, что по лемме $1 v=o\left(t^{-(\sigma+1) / \sigma} \ln t\right)$, и воспользовавшись формулой Тейлора, получим, что

$$
\begin{aligned}
& a(x)\left[\left(v+u_{N}\right)^{\sigma+1}-\left(u_{N}\right)^{\sigma+1}\right]=a(x)(\sigma+1)\left(u_{N}\right)^{\sigma} v+O\left(u_{N}^{\sigma-1} v^{2}\right) \\
& \quad=a(x) v\left[(\sigma+1) c_{0}^{\sigma} t^{-1}+(\sigma+1) \sigma c_{0}^{\sigma-1} c_{11} t^{-2} \ln t+o\left(t^{-2} \ln t\right)\right]=Q v,
\end{aligned}
$$

поэтому уравнение (20) можно записать в виде

$$
v_{t}=\Delta v-Q v+f_{N}
$$

Уравнение, которому в $\Omega \times \mathbb{R}$ удовлетворяет функция $v_{1}(x, t)=v(x, t) \zeta(t)$, где $\zeta(t)$ - гладкая класса $C^{1}$ функция, $\zeta(t)=0$ при $t<0$ и $\zeta(t)=1$ при $t>1$, имеет вид

$$
\left(v_{1}\right)_{t}=\Delta v_{1}-Q(x, t) v_{1}+F+f_{N}, \quad x \in \Omega, \quad t \in \mathbb{R}
$$

где функция $F$ финитна, при $t<0 \quad Q(x, t)=0$, при $t>1 Q(x, t)$ определяется в $(21)$, а $v_{1}$ удовлетворяет нулевому условию Неймана на гранище цилиндра $\partial \Omega \times \mathbb{R}$.

Проверим, что для уравнения (22) верны условия предложения 2 . Произвольно выбирая $\varepsilon, 0<\varepsilon<1$, из (21) получаем, что при $t \rightarrow \infty$

$$
\left(\int_{\Omega} Q^{2}(x, t) d x\right)^{1 / 2}=O\left(t^{-1}\right), \quad \int_{\Omega}-Q(x, t) d x=-\frac{\sigma+1}{\sigma} t^{-1}+o\left(t^{-1-\varepsilon}\right) .
$$

Осталось показать, что

$$
\int_{1}^{\infty} \int_{\Omega}\left|f_{N}(x, t)+F(x, t)\right|^{2} t^{2 q} d x d t<\infty
$$

при

$$
q>\frac{1}{2}+\frac{\sigma+1}{\sigma} .
$$

Так как функция $F$ финитна, то достаточно проверить сходимость интеграла

$$
\int_{1}^{\infty} \int_{\Omega}\left|f_{N}(x, t)\right|^{2} t^{2 q} d x d t<\infty
$$

В силу (12) для этого необходимо, чтобы $2 q-2 N-2 / \sigma<-1$, т.е.

$$
N>q+\frac{1}{2}-\frac{1}{\sigma}
$$

Значит, если $q=N-1 / 2+1 / \sigma-\varepsilon$, где $\varepsilon>0$ - маленькое положительное число, а $N>2+\varepsilon$, то (24) и (25) верны и, следовательно, получили, что (23) вьполнено. Поэтому из предложения 2 следует, что уравнение (22) имеет решение $v_{1}^{\prime}$, удовлетворяющее нулевым условиям Неймана, такое, что

$$
v_{1}^{\prime}=o\left(t^{1-q}\right)=o\left(t^{1-N-1 / \sigma+1 / 2+\varepsilon}\right), \quad t \rightarrow \infty .
$$


Обозначая $v_{2}=v_{1}-v_{1}^{\prime}$, находим, что $v_{2}$ удовлетворяет уравнению вида $(22)$, где функция $F$ финитна, $f_{N}=0$.

Применяя теперь предложение 1 , получаем асимптотическое представление

$$
v_{2}(x, t)=c t^{-(\sigma+1) / \sigma}+o\left(t^{-(\sigma+1) / \sigma}\right)
$$

при $t \rightarrow \infty$. Из формулы (11) следует, что

$$
u_{N}(x, t+\tau)-u_{N}(x, t)=-\frac{c_{0}}{\sigma} t^{-(\sigma+1) / \sigma} \tau+o\left(t^{-(\sigma+1) / \sigma}\right),
$$

поэтому для некоторого $\tau$ выполнено

$$
v_{2}^{\prime}=\left(u(x, t)-u_{N}(x, t+\tau)\right) \zeta_{1}(t)-v_{1}^{\prime}=o\left(t^{-(\sigma+1) / \sigma}\right), \quad t \rightarrow \infty
$$

Переход от $u_{N}(x, t) \mathrm{k} u_{N}(x, t+\tau)$ соответствует правильному выбору константы $c_{10}$.

Так как при $t \rightarrow \infty Q(x, t) \rightarrow 0$, то можно выбрать такое $t_{0}$, что для всех $t>t_{0}$ выполнено неравенство $|Q(x, t)|<\nu$, где $\nu$ определено в предложении 3. Тогда к уравнению, которому удовлетворяет функция $v_{2}^{\prime} \zeta_{2}(t)$, где $\zeta_{2}(t)$ - гладкая класса $C^{1}$ функция, $\zeta_{2}(t)=0$ при $t<t_{0}$ и $\zeta_{2}(t)=1$ при $t>t_{0}+1$, применимо предложение 3 . Поэтому существует $v_{3}$ вида (6) такая, что

$$
w=v_{2}^{\prime} \zeta_{2}-v_{3}= \begin{cases}o\left(t^{-(\sigma+1) / \sigma}\right), & t \rightarrow+\infty \\ C+o\left(e^{\alpha t}\right), & t \rightarrow-\infty\end{cases}
$$

- решение однородного уравнения (3), удовлетворяющее условию (4).

Если $C>0$, то из принципа максимума следует, что $w>0$. Покажем, что уравнение $\Delta w-Q(x, t) w-w_{t}=0$, которому удовлетворяет $w$, имеет субрешение, асимптотически эквивалентное $t^{-(\sigma+1) / \sigma}$ при $t \rightarrow+\infty$. Подберем константу $A$, функции $B(x), C(x)$ так, чтобы

$$
w_{0}=t^{-1-1 / \sigma}+A t^{-2-1 / \sigma} \ln t+B(x) t^{-2-1 / \sigma}+C(x) t^{-3-1 / \sigma} \ln t
$$

было искомым субрешением. После прямых вычислений получаем

$$
\Delta w_{0}=\Delta B t^{-2-1 / \sigma}+\Delta C t^{-3-1 / \sigma} \ln t
$$

Вычислим $\left(w_{0}\right)_{t}$ с точностью до $o\left(t^{-3-1 / \sigma} \ln t\right)$ при $t \rightarrow+\infty$ :

$$
\left(w_{0}\right)_{t}=-\left(1+\frac{1}{\sigma}\right) t^{-2-1 / \sigma}-\left(2+\frac{1}{\sigma}\right) A t^{-3-1 / \sigma} \ln t .
$$

Найдем $Q(x, t) w_{0}$, где $Q(x, t)=a(x)\left((\sigma+1) c_{0}^{\sigma} t^{-1}+(\sigma+1) \sigma c_{0}^{\sigma-1} c_{11} t^{-2} \ln t+\right.$ $\left.o\left(t^{-2} \ln t\right)\right)$ при $t \rightarrow+\infty$. Получим:

$$
\begin{aligned}
Q(x, t) w_{0}= & \frac{a(x)}{\bar{a}} \frac{\sigma+1}{\sigma} t^{-2-1 / \sigma}+\frac{a(x)}{\bar{a}} \frac{\sigma+1}{\sigma} A t^{-3-1 / \sigma} \ln t \\
& +(\sigma+1) \frac{a(x)}{\bar{a}} \frac{c_{11}}{c_{0}} t^{-3-1 / \sigma} \ln t+o\left(t^{-3-1 / \sigma} \ln t\right), \quad t \rightarrow+\infty
\end{aligned}
$$


Коэффициенты $B(x), C(x)$ будем находить из уравнений

$$
\begin{aligned}
& \Delta B=-\left(1+\frac{1}{\sigma}\right)+\frac{a(x)}{\bar{a}} \frac{\sigma+1}{\sigma} \\
& \Delta C=-\left(2+\frac{1}{\sigma}\right) A+\frac{a(x)}{\bar{a}} \frac{\sigma+1}{\sigma} A+(\sigma+1) \frac{a(x)}{\bar{a}} \frac{c_{11}}{c_{0}}+\varepsilon .
\end{aligned}
$$

Условие разрешимости уравнения для $B(x)$ выполнено, а из условия разрешимости уравнения для $C(x)$ находим, что $A=\varepsilon+(\sigma+1) c_{11} / c_{0}$. Определяя теперь функции $B(x), C(x)$ из этих уравнений, находим, что

$$
\Delta w_{0}-Q(x, t) w_{0}-\left(w_{0}\right)_{t}=\varepsilon t^{-3-1 / \sigma} \ln t+o\left(t^{-3-1 / \sigma} \ln t\right), \quad t \rightarrow+\infty,
$$

и, выбрав $\varepsilon$ положительным, получим, что $w_{0}(x, t)$ - субрешение.

Поэтому найдется такое достаточно большое $t_{1}$, что для всех $t \geqslant t_{1}$ выполнено $w>k_{1} w_{0}$ с некоторой малой положительной константой $k_{1}$, однако это противоречит тому, что $w=o\left(t^{-(\sigma+1) / \sigma}\right)$ при $t \rightarrow+\infty$. Поэтому $C=0$ и, следовательно, $w=0, v_{3}=v_{2}^{\prime} \zeta_{2}$, откуда получается, что функция

$$
v_{2}^{\prime}=\left(u(x, t)-u_{N}(x, t+\tau)\right) \zeta_{1}(t)-v_{1}^{\prime}
$$

экспоненциально убывает. Так как $v_{1}^{\prime}=o\left(t^{1-N-1 / \sigma+1 / 2+\varepsilon}\right)$, а отличие разности $v=u(x, t)-u_{N}(x, t+\tau)$ от функции $v_{1}^{\prime}$ экспоненциально мало, то $v=$ $o\left(t^{1-N-1 / \sigma+1 / 2+\varepsilon}\right)$ при $t \rightarrow+\infty$, и теорема доказана.

Приведенное рассуждение показывает, что если $\varepsilon$ выбрано меншшим чем $1 / 2$, то $u$ обладает асимптотическим разложением, совпадающим с теми слагаемыми суммы $u_{N}$, для которых $k<N$. Рассуждение применимо и для $N+1$, следовательно,

$$
u(x, t)=u_{N}(x, t)+o\left(t^{-1-1 / \sigma-N+\varepsilon}\right), \quad t \rightarrow \infty, \quad 0<\varepsilon<1 .
$$

\section{§6. Оценка разности двух положительных решений}

ТЕОРема 3. Для любых двух полоомительных решений $u(x, t)$ и $h(x, t)$ уравнения (1), удовлетворяющих условию (2), существует такая константа $\tau$, чmo

$$
u(x, t)-h(x, t+\tau)=o\left(e^{-\alpha t}\right), \quad t \rightarrow \infty,
$$

әде константа $\alpha$ не зависит от $u(x, t), h(x, t)$.

ДокАЗАТЕЛЬСтво. Сделав в уравнении (1) замену $u=v+h$, получим

$$
v_{t}=\Delta v-a(x) \frac{(v+h)^{\sigma+1}-h^{\sigma+1}}{v} v .
$$

Учитывая, что по лемме 1 имеют место соотношения

$$
\begin{gathered}
v=o\left(t^{-(\sigma+1) / \sigma} \ln t\right), \quad h^{\sigma-1} v=o\left(t^{-2} \ln t\right), \\
h^{\sigma}=c_{0}^{\sigma} t^{-1}+\sigma c_{0}^{\sigma-1} c_{11} t^{-2} \ln t+o\left(t^{-2} \ln t\right),
\end{gathered}
$$


воспользовавшись формулой Тейлора, получим

$$
\begin{aligned}
(v+h)^{\sigma+1}-h^{\sigma+1} & =h^{\sigma+1}\left(\left(1+\frac{v}{h}\right)^{\sigma+1}-1\right)=(\sigma+1) h^{\sigma} v+O\left(h^{\sigma-1} v^{2}\right) \\
& =v\left[(\sigma+1) c_{0}^{\sigma} t^{-1}+(\sigma+1) \sigma c_{0}^{\sigma-1} c_{11} t^{-2} \ln t+o\left(t^{-2} \ln t\right)\right] .
\end{aligned}
$$

Следовательно, уравнение (26) можно записать в виде

$$
v_{t}=\Delta v-a(x)\left(c_{0}^{\sigma}(\sigma+1) t^{-1}+(\sigma+1) \sigma c_{0}^{\sigma-1} c_{11} t^{-2} \ln t+o\left(t^{-2} \ln t\right)\right) v .
$$

Пусть $\zeta_{1}(t)$ - гладкая класса $C^{1}$ функция, $\zeta_{1}(t)=0$ при $t<0$ и $\zeta_{1}(t)=1$ при $t>1$. Тогда $v_{1}(x, t)=v(x, t) \zeta_{1}(t)$ является удовлетворяюшим нулевому условию Неймана на $\partial \Omega \times \mathbb{R}$ решением уравнения

$$
\left(v_{1}\right)_{t}=\Delta v_{1}-Q(x, t) v_{1}+F, \quad x \in \Omega, \quad t \in \mathbb{R}
$$

где функция $F$ финитна по $t, Q(x, t)=0, t<0$, и

$$
Q(x, t)=a(x)\left(c_{0}^{\sigma}(\sigma+1) t^{-1}+(\sigma+1) \sigma c_{0}^{\sigma-1} c_{11} t^{-2} \ln t+o\left(t^{-2} \ln t\right)\right), \quad t>1 .
$$

Следовательно, для любого $\varepsilon, 0<\varepsilon<1$, при $t \rightarrow \infty$

$$
\begin{gathered}
\left(\int_{\Omega} Q^{2}(x, t) d x\right)^{1 / 2}=O\left(t^{-1}\right) \\
\int_{\Omega}-Q(x, t) d x=-\bar{a} c_{0}^{\sigma}(\sigma+1) t^{-1}+o\left(t^{-1-\varepsilon}\right)=-\frac{\sigma+1}{\sigma} t^{-1}+o\left(t^{-1-\varepsilon}\right) .
\end{gathered}
$$

Поскольку функция $F$ финитна по $t$, то для любого $q$ сходится интеграл

$$
\int_{1}^{\infty} \int_{\Omega}|F(x, t)|^{2} t^{2 q} d x d t<\infty .
$$

Таким образом, выполнены условия предложения 1. Из предложения 1 получаем, что

$$
v_{1}(x, t)=c t^{-(\sigma+1) / \sigma}+o\left(t^{-(\sigma+1) / \sigma}\right), \quad t \rightarrow \infty .
$$

Из теоремы 2 следует, что $h(x, t+\tau)-h(x, t)=-\frac{c_{0}}{\sigma} t^{-(\sigma+1) / \sigma} \tau+o\left(t^{-(\sigma+1) / \sigma}\right)$, и поэтому можно подобрать $\tau$ так, что

$$
v_{2}(x, t)=(u(x, t)-h(x, t+\tau)) \zeta_{1}(t)=o\left(t^{-(\sigma+1) / \sigma}\right), \quad t \rightarrow \infty .
$$

Поскольку $Q(x, t) \rightarrow 0$ при $t \rightarrow \infty$, можно выбрать такое $t_{0}$, что для всех $t>t_{0}$ выполнено неравенство $|Q(x, t)|<\nu$, где $\nu$ определено в предложении 3. Для уравнения, которому удовлетворяет функция $v_{2} \zeta_{2}(t)$, где $\zeta_{2}(t)$ - гладкая класса $C^{1}$ функция, $\zeta_{2}(t)=0$ при $t<t_{0}$ и $\zeta_{2}(t)=1$ при $t>t_{0}+1$, выполнены условия предложения 3 . Поэтому найдется $v_{3}$ вида $(6)$ такая, что

$$
w=v_{2} \zeta_{2}-v_{3}= \begin{cases}o\left(t^{-(\sigma+1) / \sigma}\right), & t \rightarrow+\infty \\ C+o\left(e^{\alpha t}\right), & t \rightarrow-\infty\end{cases}
$$

- решение однородного уравнения вида (3), удовлетворяющее условию (4). 
Если $C>0$, то из принципа максимума заключаем, что $w>0$. Рассуждением, аналогичным проведенному при доказательстве теоремы 2 , можно показать, что это уравнение имеет субрешение $w_{0}$, асимптотически эквивалентное $t^{-(\sigma+1) / \sigma}$ при $t \rightarrow+\infty$. Так как при достаточно большом $t_{1}$ функция $w_{0}(x, t)$ положительна, то в силу положительности $w\left(x, t_{1}\right)$ при достаточно малой константе $k_{1}$ из принципа максимума получим, что $w>k_{1} w_{0}$ при всех $t>t_{1}$. Это противоречит тому, что $w=o\left(t^{-(\sigma+1) / \sigma}\right)$ при $t \rightarrow+\infty$. Аналогично, допущение $C<0$ приводит к противоречию. Поэтому $C=0$ и, следовательно, $w=0$, т.е. $v_{3}=v_{2} \zeta_{2}$. Таким образом, разность $u(x, t)-h(x, t+\tau)$ совпадает при больших $t c v_{3}$, а следовательно, экспоненциально убывает.

ЗАмЕчАниЕ. Если $a(x)$ - не обязательно положительная ограниченная измеримая функция такая, что $\bar{a}>0$, то для стремяшихся к нулю решений в $\Pi_{0}$ уравнения (1), удовлетворяюших условию (2), будут выполнены теоремы 1-3.

Автор выражает глубокую благодарность В.А. Кондратьеву, под руководством которого были получены эти результаты.

\section{Список литературы}

1. Ладыженскал О.А., Солонников В. А., Уральцева Н. Н. Линейные и квазилинейные уравнения параболического типа. М.: Наука, 1967.

2. Baras $P$., Veron L. Comportement asymptotique de la solution d'une équation d'évolution semi-linéaire de la chaleur // Comm. Partial Differential Equations. 1979. V. 4. P. 795-807.

3. Kondratiev V. A., Veron L. Asymptotic behaviour of solutions of some nonlinear parabolic or elliptic equations // Asymptot. Anal. 1997. V. 14. № 2. P. 117-156.

4. Арефьев В.Н., Кондратьев В. А. Асимптотическое поведение решений второй краевой задачи для нелинейных параболических уравнений // Дифференц. уравнения. 1993. Т. 29. № 12. C. 2104-2116.

5. Багиров Л.А., Кондратьев В.А. Об асимптотических свойствах решений уравнения диффузии // Труды сем. им. И. Г. Петровского. Т. 22. М.: Изд-во МГУ, 2002. С. 37-70.

6. Агранович М. С., Вишик М. И. Эллиптические задачи с параметром и параболические задачи общего вида // УМН. 1964. Т. 19. №3. С. 53-161.

7. Гохберг И. Ц., Сигал Е. И. Операторное обобщение теоремы о логарифмическом вычете и теоремы Руше // Матем. сб. 1971. Т. 84(126). № 4. С. 607-629.

8. Agmon S., Nirenberg L. Properties of solutions of ordinary differential equations in Banach space // Comm. Pure Appl. Math. 1963. V. 16. P. 121-239.

Московский государственньй университет

им. М.В. Ломоносова

Поступила в редакцию

E-mail: filimi@yandex.ru

07.07.2003 